\title{
CONFIGURATION SPACES AND THE SPACE OF RATIONAL CURVES ON A TORIC VARIETY
}

\author{
M. A. GUEST
}

\begin{abstract}
The space of holomorphic maps from $S^{2}$ to a complex algebraic variety $X$, i.e. the space of parametrized rational curves on $X$, arises in several areas of geometry. It is a well known problem to determine an integer $n(D)$ such that the inclusion of this space in the corresponding space of continuous maps induces isomorphisms of homotopy groups up to dimension $n(D)$, where $D$ denotes the homotopy class of the maps. The solution to this problem is known for an important but special class of varieties, the generalized flag manifolds: such an integer may be computed, and $n(D) \rightarrow \infty$ as $D \rightarrow \infty$. We consider the problem for another class of varieties, namely, toric varieties. For smooth toric varieties and certain singular ones, $n(D)$ may be computed, and $n(D) \rightarrow \infty$ as $D \rightarrow \infty$. For other singular toric varieties, however, it turns out that $n(D)$ cannot always be made arbitrarily large by a suitable choice of $D$.
\end{abstract}

The space of all holomorphic maps from the Riemann sphere $S^{2}=\mathbb{C} \cup \infty$ to a complex projective variety $X$ will be denoted by $\operatorname{Hol}\left(S^{2}, X\right)$. (In this context, "holomorphic" means the same as "algebraic" or "rational", but we use the first term so as not to preclude more general situations later.) The global topological properties of $\operatorname{Hol}\left(S^{2}, X\right)$ are relevant to various problems in algebraic topology, algebraic geometry, differential geometry, and mathematical physics.

When $X=S^{2}, \operatorname{Hol}\left(S^{2}, X\right)$ is just the space of rational functions - topologized as a subspace of the $\operatorname{space} \operatorname{Map}\left(S^{2}, X\right)$ of continuous (or smooth) maps. It is elementary that each connected component of $\mathrm{Hol}\left(S^{2}, S^{2}\right)$ is indexed by a non-negative integer (the degree, or the number of poles, of a map). The next topological invariant, the fundamental group, was computed independently by Epshtein ([Ep]) and Jones (see $\S 6$ of [Se]): if $\mathrm{Hol}_{d}\left(S^{2}, S^{2}\right)$ denotes the maps of degree $d$ and $\operatorname{Hol}_{d}^{*}\left(S^{2}, S^{2}\right)$ the maps of degree $d$ which fix the point $\infty$, then $\pi_{1} \operatorname{Hol}_{d}\left(S^{2}, S^{2}\right)$ is cyclic of order $2 d$, and $\pi_{1} \operatorname{Hol}_{d}^{*}\left(S^{2}, S^{2}\right) \cong \mathbb{Z}$. Now, it happens that these agree with the fundamental groups of the corresponding spaces $\operatorname{Map}_{d}\left(S^{2}, S^{2}\right), \operatorname{Map}_{d}^{*}\left(S^{2}, S^{2}\right)$. An "explanation" for this coincidence is provided by Morse theory, for $\operatorname{Hol}_{d}\left(S^{2}, S^{2}\right)$ is the space of absolute minima of the energy functional $E: \operatorname{Map}_{d}\left(S^{2}, S^{2}\right) \rightarrow \mathbb{R}, f \mapsto \int_{S^{2}}|d f|^{2}$ (see [EL]). Thus (cf. $\S 22$ of [Mi]), one expects the space of holomorphic maps to have the same homotopy groups as the space of continuous maps, up to some dimension. (This applies more generally to $\operatorname{Hol}\left(S^{2}, X\right)$ for any Kähler manifold $X$ and is therefore of interest from the point of view of harmonic maps or minimal immersions of $S^{2}$ into such spaces.) Although Morse theory does not apply naively to the energy functional here, such a "comparison theorem" was estab-

Received by the editors November 15, 1993.

1991 Mathematics Subject Classification. Primary 55P99, 14 M25. 
lished without appealing to Morse theory by Segal, who showed in [Se] that the inclusions

$$
\operatorname{Hol}_{d}\left(S^{2}, S^{2}\right) \rightarrow \operatorname{Map}_{d}\left(S^{2}, S^{2}\right) \quad \text { and } \quad \operatorname{Hol}_{d}^{*}\left(S^{2}, S^{2}\right) \rightarrow \operatorname{Map}_{d}^{*}\left(S^{2}, S^{2}\right)
$$

induce isomorphisms of homotopy groups $\pi_{i}$ for $0 \leq i<d$. In particular, these maps also induce isomorphisms in integral homology groups in the same range. From the point of view of algebraic topology, this leads to an interesting development (see [CCMM], and also [CS, Va]), because the homology of $\operatorname{Map}_{d}^{*}\left(S^{2}, S^{2}\right)=\Omega_{d}^{2} S^{2}$ is well known. This homology has a natural algebraic filtration, and one of the main results of [CCMM] is that the filtration is realized geometrically by the spaces $\operatorname{Hol}_{d}^{*}\left(S^{2}, S^{2}\right)$. It should be mentioned that Segal's result is also relevant to, and was motivated partly by, a problem in control theory (cf. [BD]).

As predicted in [Se], the comparison theorem has been extended to the inclusion $\operatorname{Hol}_{d}^{*}\left(S^{2}, X\right) \rightarrow \operatorname{Map}_{d}^{*}\left(S^{2}, X\right)$ when $X$ is any generalized flag manifold (i.e. $X=G^{\mathbb{C}} / P$ where $G^{\mathbb{C}}$ is a complex semisimple Lie group and $P$ is a parabolic subgroup). This was carried out in [Gu1, Ki1, Gr, MM1, MM2, BHMM2]. It has even been extended to the case where $X=\Omega G([\mathrm{Gr}, \mathrm{Ta}$, BHMM1, $\mathrm{Ki2}$ ]; see also [Gu2]), which is an infinite dimensional generalized flag manifold. Apart from its relevance to control theory and to harmonic maps, this result is of interest from the point of view of Yang-Mills theory, as $\operatorname{Hol}^{*}\left(S^{2}, G^{\mathbb{C}} / P\right)$ may be interpreted as a moduli space of monopoles. The space $\operatorname{Hol}^{*}\left(S^{2}, \Omega G\right)$ may be interpreted as a moduli space of $G$-instantons over $S^{4}$, and the comparison theorem in this case was conjectured by Atiyah and Jones $[\mathrm{AJ}]$ in the early days of mathematical Yang-Mills theory.

With the notable exception of [Se], the work in proving the comparison theorem for $X=G^{\mathrm{C}} / P$ employed traditional methods of algebraic topology and relied either on the results of [Se] or on knowing beforehand the homology of the spaces of continuous maps. It is natural to ask whether the theorem can be extended to larger classes of complex manifolds (or varieties or analytic spaces), and it is natural to ask for a more conceptual proof. There are some obvious limitations; for example, there may be no (or only finitely many) non-constant holomorphic maps from $S^{2}$ to $X$. In the case where $\operatorname{Hol}\left(S^{2}, X\right)$ is large, a conceptual (Morse theoretic) proof may ultimately be provided by the analytical methods of Donaldson, Taubes, and Uhlenbeck (cf. $\S 4$ of [Uh]) or by the Gromov-Floer theory developed in [CJS]. So far, however, this goal has proved elusive.

In view of this, it is of interest to investigate concrete examples beyond the class of generalized flag manifolds, and we shall do this by establishing the comparison theorem for a large class of toric varieties. Our method is also based on [Se], but it uses the ideas rather than the results of [Se] and is therefore quite self-contained. Moreover, our method represents a technical simplification of the method of [Se] (which in turn is simpler than the more traditional methods referred to above). It also makes explicit the fundamental idea that the functor $\mathbb{C} \mapsto \pi_{i} \operatorname{Hol}(\mathbb{C} \cup \infty, X)$ behaves like a generalization of a homology functor.

Before stating our results, we recall briefly the definition of a toric variety: a toric variety is an irreducible normal algebraic variety $X$ which admits an (algebraic) action of an algebraic torus $T^{\mathbb{C}}=\mathbb{C}^{*} \times \cdots \times \mathbb{C}^{*}$ such that the orbit $T^{\mathbb{C}} \cdot *$ of some point $* \in X$ is a densely embedded copy of $T^{\mathbb{C}}$. Such varieties 
were considered in the 1970s in connection with compactifications in algebraic geometry, but they have only recently been introduced to wider audiences [Od, $\mathrm{Fu}$. Their attractiveness derives from the fact that they are amenable to study by combinatorial methods. This is because a toric variety $X$ is characterized by its "fan" $\Delta$, a collection of convex cones in $\mathbb{R}^{k}$ (where $k=\operatorname{dim}_{\mathbb{C}} T^{\mathrm{C}}$ ). In the case of projective algebraic varieties, toric varieties are characterized as being definable by means of "monomial equations".

Our first result gives a description of $\mathrm{Hol}^{*}\left(S^{2}, X\right)$ as a "labelled configuration space". (The basepoint condition here is taken to be $f(\infty)=*$.) To establish notation, let $Q(\mathbb{C} ; M)$ denote the space of configurations of distinct points in $\mathbb{C}$ which have labels in a partial monoid $M$. This space is topologized in such a way that two labelled points $z_{1}, z_{2}$ are allowed to collide if the sum of their labels $m_{1}, m_{2}$ is defined in $M$ (the result of such a collision would be a new point with label $m_{1}+m_{2}$ ); otherwise the collision is prohibited. To a fan $\Delta$ we associate a partial monoid $M_{\Delta}$, which is a certain subset of $\operatorname{Hom}(G, \mathbb{Z})$, the dual of the group $G$ of $T^{\mathbb{C}}$-equivariant Cartier divisors on $X$. There is a homomorphism $h: \pi_{2} X \rightarrow \operatorname{Hom}(G, \mathbb{Z})$.

Proposition 1. Let $D \in \pi_{2} X$. Then the space $\operatorname{Hol}_{D}^{*}\left(S^{2}, X\right)$ is diffeomorphic to the configuration space

$$
Q_{D}\left(\mathbb{C} ; M_{\Delta}\right)=\left\{\left\{\left(z_{i}, m_{i}\right)\right\}_{i} \in Q\left(\mathbb{C} ; M_{\Delta}\right) \mid \sum_{i} m_{i}=h(D)\right\} .
$$

This generalizes the fact that a basepoint-preserving rational function is determined by its poles and zeros. Similar configuration spaces have appeared in algebraic topology since the work of Dold and Thom [DT] on symmetric products. Indeed, the symmetric product $\operatorname{Sp}^{k}(U)$ of a space $U$ is the simplest example of this type; it may be considered as a subspace of the configuration space $Q\left(U ; M_{k}\right)$, where $M_{k}$ is the partial monoid $\{1,2, \ldots, k\}$. The main result of [DT] is that the functor $U \mapsto \pi_{i} \operatorname{Sp}^{k}(U)$ agrees with the ordinary homology functor $H_{i}$, for all $i$ up to some dimension, on nice spaces $U$. The same method shows that the functor $U \mapsto \pi_{i} Q_{D}\left(U ; M_{\Delta}\right)$ satisfies the axioms for a generalized homology theory, for a range of values of $i$, on an appropriate category.

In the case of a smooth toric variety $X, \pi_{2} X$ may be identified naturally with a subgroup of $\mathbb{Z}^{u}$, where $u$ is the number of codimension-one orbits of $T^{\mathrm{C}}$ in $X$, so we may write $D=\left(d_{1}, \ldots, d_{u}\right)$. The integer $d_{i}$ represents the intersection number of the map with the closure of the $i$-th orbit. We then have:

Theorem 2. Let $X$ be a smooth toric variety. Then the inclusion $\operatorname{Hol}_{D}^{*}\left(S^{2}, X\right) \rightarrow$ $\operatorname{Map}_{D}^{*}\left(S^{2}, X\right)$ induces isomorphisms of homotopy groups $\pi_{i}$ for $0 \leq i<$ $\min \left\{d_{1}, \ldots, d_{u}\right\}$.

The proof has two main ingredients. One is similar to an argument of [Se]: it uses the homology-like properties of the functor $\mathbb{C} \mapsto \pi_{i} Q_{D}\left(\mathbb{C} \cup \infty ; M_{\Delta}\right)$ to show that one obtains a homotopy equivalence in the limit $D \rightarrow \infty$. The other ingredient is the fact that there are "stabilization maps" $Q_{d_{1}, \ldots, d_{u}}\left(\mathbb{C} ; M_{\Delta}\right) \rightarrow$ $Q_{d_{1}^{\prime}, \ldots, d_{u}^{\prime}}\left(\mathbb{C} ; M_{\Delta}\right)$ (where $\left.d_{i}^{\prime} \geq d_{i}\right)$ which induce isomorphisms of homotopy groups up to dimension $\min \left\{d_{1}, \ldots, d_{u}\right\}$. Here the method differs significantly 
from that of [Se]; it was introduced in [GKY]. The idea is to reduce to the corresponding statement for symmetric products, which is elementary. This has the advantage that the passage from homology to homotopy is easier than in [Se].

When $X$ is singular, the situation is considerably more complicated. Nevertheless, we can give a method for determining the extent to which the comparison theorem holds, based upon the choice of a toric resolution $\hat{X}$ of $X$. Such a resolution may be obtained by subdividing the fan $\Delta$ by "inserting rays". (The precise meaning of this is explained in $\S 2.7$ of [Fu].) The simplest application of our method gives:

Theorem 3. Let $X$ be a toric variety. Assume that a toric resolution $\hat{X}$ of $X$ may be found by inserting a single ray in the fan $\Delta$ of $X$. Then the inclusion $\operatorname{Hol}_{D}^{*}\left(S^{2}, X\right) \rightarrow \operatorname{Map}_{D}^{*}\left(S^{2}, X\right)$ induces isomorphisms of homotopy groups $\pi_{i}$ for $0 \leq i<n(D)$, where $n(D) \rightarrow \infty$ as $D \rightarrow \infty$.

In the situation of Theorem 3, we can in fact give an explicit formula for the integer $n(D)$, but we are not able to give a formula in the case of a general toric variety, partly because the integer $n(D)$ depends on the choice of resolution (which is far from unique). However, it is possible to use this method to determine $n(D)$ in concrete examples, two of which are given below.

Example 1. (See [Gu3].) The subvariety $X$ of $\mathbb{C} P^{3}$ defined by the monomial equation $z_{2}^{2}=z_{1} z_{3}$ has only one singular point, which may be resolved by inserting a single ray in the fan of $X$. Here, $\pi_{2} X \cong \mathbb{Z}$, and the method of Theorem 3 shows that the inclusion $\operatorname{Hol}_{d}^{*}\left(S^{2}, X\right) \rightarrow \operatorname{Map}_{d}^{*}\left(S^{2}, X\right)$ induces isomorphisms of homotopy groups $\pi_{i}$ for $0 \leq i<[d / 3]$.

Example 2. The weighted projective space $X=P(1,2,3)$ is by definition the quotient space of $\mathbb{C}^{3}-\{0\}$ by the action $u \cdot\left[z_{0} ; z_{1} ; z_{2}\right]=\left[u z_{0} ; u^{2} z_{1} ; u^{3} z_{2}\right]$ of $\mathbb{C}^{*}$. There are two singular points, which may be resolved by inserting three rays. Again, $\pi_{2} X \cong \mathbb{Z}$, and three applications of the above method lead to the conclusion that the inclusion $\operatorname{Hol}_{d}^{*}\left(S^{2}, X\right) \rightarrow \operatorname{Map}_{d}^{*}\left(S^{2}, X\right)$ induces isomorphisms of homotopy groups up to dimension $\left[\frac{2}{5} d\right]$.

We do not claim, however, that the method always leads to an integer $n(D)$ such that $n(D) \rightarrow \infty$ as $D \rightarrow \infty$. Another source of trouble is the choice of basepoint in $X$ - this is relevant as toric varieties are not in general homogeneous spaces. So far we have assumed that the basepoint of $X$ is a point $*$ such that the closure of $T^{\mathbb{C}} \cdot *$ is $X$. If a different basepoint (or no basepoint at all) is used, then Theorem 2 remains valid with possibly a smaller range of homotopy groups but Theorem 3 may fail completely. This is illustrated by the variety of Example 1 above, where the results for all basepoints were analyzed in [Gu3]. Nevertheless, it turns out that in all our counterexamples to (the conclusion of) Theorem 3, a modification of Theorem 3 holds in which $\mathrm{Hol}_{D}^{*}\left(S^{2}, X\right)$ is replaced by an appropriate subspace. Full details will appear elsewhere.

The author is greatly indebted to Andrzej Kozlowski and Kohhei Yamaguchi for their generous encouragement and collaboration. 


\section{REFERENCES}

[AJ] M. F. Atiyah and J. D. S. Jones, Topological aspects of Yang-Mills theory, Comm. Math. Phys. 61 (1978), 97-118.

[BD] C. I. Byrnes and T. Duncan, On certain topological invariants arising in system theory, New Directions in Applied Mathematics, Springer, New York, 1981, pp. 29-71.

[BHMM1] C. P. Boyer, J. C. Hurtubise, B. M. Mann, and R. J. Milgram, The topology of instanton moduli spaces. I: The Atiyah-Jones conjecture, Ann. of Math. (2) 137 (1993), 561-609.

[BHMM2] —_, The topology of the space of rational maps into generalized flag manifolds, preprint.

[CCMM] F. R. Cohen, R. L. Cohen, B. M. Mann, and R. J. Milgram, The topology of rational functions and divisors of surfaces, Acta Math. 166 (1991), 163-221.

[CJS] R. L. Cohen, J. D. S. Jones, and G. B. Segal, Morse theory and classifying spaces, preprint.

[CS] R. L. Cohen and D. H. Shimamoto, Rational functions, labelled configurations, and Hilbert schemes, J. London Math. Soc. (2) 43 (1991), 509-528.

[DT] A. Dold and R. Thom, Quasifaserungen und unendliche symmetrische Produkte, Ann. of Math. (2) 67 (1958), 239-281.

[EL] J. Eells and L. Lemaire, A report on harmonic maps, Bull. London Math. Soc. 10 (1978), $1-68$.

[Ep] S. I. Epshtein, Fundamental groups of spaces of coprime polynomials, Funct. Anal. Appl. 7 (1973), 82-83.

[Fu] W. Fulton, Introduction to toric varieties, Ann. of Math. Stud., vol. 131, Princeton Univ. Press, Princeton, NJ, 1993.

[GKY] M. A. Guest, A. Kozlowski, and K. Yamaguchi, The topology of spaces of coprime polynomials, Math. Z. (to appear).

[Gr] J. Graveson, On the topology of spaces of holomorphic maps, Acta Math. 162 (1989), 247-286.

[Gu1] M. A. Guest, Topology of the space of absolute minima of the energy functional, Amer J. Math. 106 (1984), 21-42.

[Gu2] _ Instantons, rational maps, and harmonic maps, Mat. Contemp. 2 (1992), 113 155.

[Gu3] On the space of holomorphic maps from the Riemann sphere to the quadric cone, Quart. J. Math. Oxford Ser. (2) 45 (1994), 57-75.

[Ki1] F. C. Kirwan, On spaces of maps from Riemann surfaces to Grassmannians and applications to the cohomology of moduli of vector bundles, Ark. Mat. 24 (1986), 221-275.

[Ki2] Geometric invariant theory and the Atiyah-Jones conjecture, Proc. Sophus Lie Memorial Conf., Oslo, 1992 (to appear).

[Mi] J. Milnor, Morse theory, Ann. of Math. Stud., vol. 51, Princeton Univ. Press, Princeton, NJ, 1963.

[MM1] B. M. Mann and R. J. Milgram, Some spaces of holomorphic maps to complex Grassmann manifolds, J. Differential Geom. 33 (1991), 301-324.

[MM2] On the geometry of $S U(n)$-monopoles and holomorphic maps to flag manifolds, J. Differential Geom. 38 (1993), 39-103.

[Od] T. Oda, Convex bodies and algebraic geometry: An introduction to the theory of toric varieties, Springer, Berlin, 1988.

[Se] G. B. Segal, The topology of spaces of rational functions, Acta Math. 143 (1979), 39-72.

[Ta] C. H. Taubes, The stable topology of self dual moduli spaces, J. Differential Geom. 29 (1989), 163-230. 
[Uh] K. Uhlenbeck, Applications of non-linear analysis in topology, Proc. Internat. Cong. Math., Kyoto, 1990, Springer, Tokyo, 1991, pp. 261-279.

[Va] V. A. Vassiliev, Complements of discriminants of smooth maps: Topology and applications, Transl. Math. Monographs, vol. 98, Amer. Math. Soc., Providence, 1992.

Department of MAThematics, University of Rochester, Rochester, New York 14627

E-mail address: gues@db1.cc.rochester.edu 